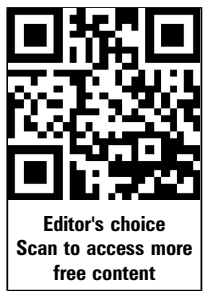

\title{
Bronchoscopic transparenchymal nodule access (BTPNA): first in human trial of a novel procedure for sampling solitary pulmonary nodules
}

\author{
Felix JF Herth, ${ }^{1,2}$ Ralf Eberhardt, 1,2 Daniel Sterman, ${ }^{3}$ Gerard A Silvestri, ${ }^{4}$ \\ Hans Hoffmann, ${ }^{5}$ Pallav L Shah ${ }^{6,7,8}$
}

'Department of Pneumology and Respiratory Care Medicine, Thoraxklinik, University of Heidelberg, Heidelberg, Germany

${ }^{2}$ Translational Lung Research Center, Heidelberg, Germany ${ }^{3}$ Department of Pulmonary Medicine, University of Pennsylvania Medical Center, Philadelphia, Pennsylvania, USA

${ }^{4}$ Department of Pulmonary Medicine, Medical University of South Carolina, Charleston, South Carolina, USA ${ }^{5}$ Department of Thoracic Surgery, Thoraxklinik, University of Heidelberg, Heidelberg, Germany

${ }^{6}$ The NIHR Respiratory Biomedical Research Unit at Royal Brompton and Harefield NHS Foundation Trust and Imperial College, London, UK ${ }^{7}$ Chelsea \& Westminster Hospital, London, UK ${ }^{8}$ National Heart \& Lung Institute, Imperial College, London, UK

\section{Correspondence to} Professor Felix JF Herth, Department of Pneumology and Respiratory Care Medicine, Thoraxklinik, University of Heidelberg, Amalienstr, 5 Heidelberg D-69126, Germany; Felix.Herth@med.uniheidelberg.de

Received 22 August 2014 Accepted 2 February 2015 Published Online First

6 March 2015

\section{ABSTRACT}

Introduction The promise of benefits from lung cancer screening is tempered by the false positive rate and the need to perform diagnostic procedures to determine the aetiology of the solitary pulmonary nodules (SPN) identified. We have developed a novel procedure which allows sampling of SPNs via a transparenchymal approach, and report the results from this as a first in human trial.

Methods This study was a prospective single-arm interventional study. We recruited patients with a SPN detected on CT imaging, which was suspicious for lung cancer, who were suitable for surgical resection. Using the subject's CT, an optimal airway wall point of entry (POE), and an avascular path through lung tissue from the POE to the SPN was calculated. A tunnel tract was created from the POE to the nodule using a set of catheter-based tools under fused fluoroscopy guidance. The patients proceeded to surgical resection immediately after the biopsy. The participants were followed-up for 6 months after the procedure. The primary endpoint of the study was to evaluate the feasibility to access and biopsy the nodule.

Results Twelve patients were recruited, and a tunnel pathway created in 10 patients. There were no adverse events during the procedures. Adequate biopsies were obtained from 10 patients (83\%), which correlated with the histological findings from the surgical resection. Inspection of the resected lobes did not raise any safety concerns and indicated appropriately tunnelled pathways to the nodule.

Conclusions This first in human study demonstrates that bronchoscopic transparenchymal access of SPNs is feasible.

Trial registration number NCT02130115.

\section{INTRODUCTION}

Lung cancer remains a challenging disease despite clear knowledge of its aetiology. In Europe, there were 400000 people diagnosed with lung cancer, and approximately 350000 expected deaths in $2010 .^{1}$ Five-year survival remains poor at $9.0 \%$. On a global basis, the 2012 statistics suggested that lung cancer was responsible for 1.59 million deaths. ${ }^{2}$ The problem is compounded as most lung cancers are diagnosed late in their natural history, and about $75 \%$ of patients have advanced disease at the time of diagnosis. ${ }^{3}$ Hence, identification of patients with lung cancer at the earliest stage is vital if outcomes

\section{Key messages}

What is the key question?

- As current diagnostic techniques for peripheral nodules detected by CT screening have particular risks and limitations, we have evaluated the safety and feasibility of a new transpulmonary approach to sample pulmonary nodules.

\section{What is the bottom line?}

- Bronchoscopic transparenchymal nodule access is a novel technique for sampling peripheral lung nodules that is not dependent on an airway leading into the nodule.

\section{Why read on?}

- This first in human study has demonstrated that it is a safe approach that is technically feasible with a high success rate.

for lung cancer are to be improved. Results from the National Lung Screening Trial have been very encouraging and have suggested a $20 \%$ reduction in lung cancer mortality in the screened group. ${ }^{4}$ However, approximately $25 \%$ of the scans demonstrated a nodule, of which most $(96.4 \%)$ were nonmalignant. Furthermore, 713 (4\%) of participants required a surgical procedure to establish a diagnosis. This was associated with a significant morbidity, as 165 subjects (23\% of those undergoing surgery) had at least one complication and there were five deaths. Increased use of surgery for the management of screen-detected nodules shifts the value of screening due to the cost implications and risks of surgical procedures. $^{5}$ A recent meta-analysis of 39 clinical studies evaluating a variety of bronchoscopic techniques with over 3000 solitary pulmonary nodules (SPN) sampled demonstrated a wide diagnostic range from $18 \%$ to $75 \% .^{6}$ Transthoracic procedures have a greater accuracy but with higher complication rates, especially pneumothoraces that occur in $15 \%$ of cases. ${ }^{7}$

Several bronchoscopic approaches exist; standard bronchoscopy with transbronchial biopsy under fluoroscopic guidance, ${ }^{8}$ ultrathin bronchoscopy, virtual bronchoscopy-guided biopsy, ${ }^{10}$ radial endobronchial ultrasound-guided biopsy, ${ }^{11}$ electromagnetic
Eberhardt R, Sterman $D_{1}$ $326-332$ 
navigation-guided biopsy, ${ }^{12}$ and even combinations of these techniques. ${ }^{13}$ The vast majority of the data originates from single centres, and the yield ranges from $67 \%$ to $73 \%$. The yield is dependent on the size of the pulmonary nodule and distance from the hilum. The diagnostic rate for nodules smaller than $20 \mathrm{~mm}$ is much lower $(46 \%) .{ }^{14}$ Some nodules are eccentrically positioned and may not have an airway directly leading to them. ${ }^{15}$ Hence, it may not be possible to approach the nodule via the bronchial tree. Furthermore, navigation and manoeuvring through several subsegments is challenging. Consequently, the yield decreases with each successive branching beyond fourth-generation bronchial segments.

We have developed a novel bronchoscopic technique for accessing nodules through a transparenchymal approach which is not dependent on the need to have an airway leading into the lesion. We report the feasibility and safety of the Bronchoscopic Trans-Parenchymal Nodule Access (BTPNA) procedure in this first in human study.

\section{METHODS}

\section{Study design and participants}

This study was a prospective single-arm interventional study. This procedure was developed for sampling SPNs, which are defined as lesions of up to $30 \mathrm{~mm}$ diameter. However, for safety reasons, as this was a first in human study, we recruited patients with lesions of up to $40 \mathrm{~mm}$ diameter from suspected lung cancer or metastatic disease, who were suitable for surgical resection. For simplicity, we have used the term SPN throughout the manuscript. The SPNs were required to be located in the pulmonary parenchyma at least $10 \mathrm{~mm}$ away from the pleural surface. Participants were required to fulfil all the inclusion criteria in the absence of any exclusion criteria (see box 1), and provide written informed consent.

\section{Planning stage}

Computerised tomograms from eligible subjects were uploaded onto the Archimedes Virtual Bronchoscopy Navigation (VBN) System (Broncus Medical, Mountain View, California, USA) which is a software system that reconstructs the CT data into a 3D model, including bronchial airways, vascular structures, ribs and lungs, and provides features to allow the user to mark and segment a target, that is, a suspicious SPN. The system calculates two suitable point of entry (POE) locations with straight line, vessel-free access to the SPN, as well as bronchoscopy paths for guiding the user's bronchoscope to the POE locations. Additionally, the Archimedes System displays the tunnel path from the POE on the airway wall to the SPN. The user is allowed to fine-tune the POE location using a 'Virtual Doppler' tool, which enables the user to inspect for presence of vessels outside of the airway wall in the virtual bronchoscopy view. Users are also able to visualise the blood vessels during the virtual bronchoscopy review of the path to the selected POE, which is painted as a target on the airway wall. A cylindrical icon on the POE describes the orientation of the bronchoscope. The bronchial diameter, bronchoscopy path distance, tunnel length to the front of the target, target size, and distance from the back of the target to the nearest pleura location are all provided in the virtual bronchoscopy view. During the procedure, the Archimedes System integrates real-time fluoroscopy data with the 3D CT data to guide a sheath from the POE on the airway wall through the lung parenchyma directly to the SPN.

\section{Procedure}

The bronchoscopic procedure was performed under general anaesthesia and muscle paralysis. The procedure was performed in the operating room as a precaution in the event of a serious event such as a major haemorrhage. Two procedures were performed by FJFH on a simulation model prior to the first patient procedure. As a further precautionary measure, a balloon blocker (Cohen Balloon, Cook Medical, Ireland) was placed at the main stem of the bronchus leading to the lobe where the nodule was located. The balloon was inflated to check adequate positioning and the volume of air required for correct inflation of the spherical balloon. ${ }^{16}$ The balloon blocker was then locked in position and the balloon deflated. A standard bronchoscopy was also performed to ensure that there were no endobronchial lesions.

\section{Box 1 Enrolment criteria}

Inclusion criteria

- Subjects suitable for surgery, aged 21 years-75 years

- Nodule due to suspected lung cancer or metastatic disease

- Nodule measuring greater than $10 \mathrm{~mm}$ and up to $40 \mathrm{~mm}$ in largest dimension

- No known endobronchial tumour

- Nodule located anywhere in parenchymal tissue $>1 \mathrm{~cm}$ from pleura

- Nodule accessible bronchoscopically through a point of entry on planning CT reconstruction

- Subject able to understand study requirements

- Subject able to provide signed consent

Exclusion Criteria

- An inability to tolerate bronchoscopy under heavy sedation or general anaesthesia

- Long-term use of anticoagulants (eg, heparin, warfarin) or antiplatelet agents (eg, aspirin, clopidogrel) which cannot be discontinued

- Uncorrectable coagulopathy

- Platelet dysfunction or platelet count $<100 \times 10$

- History of major bleeding with bronchoscopy

- Pulmonary hypertension with mean PAP > $25 \mathrm{~mm}$

- Moderate-to-severe pulmonary fibrosis

- Moderate-to-severe emphysema or COPD with FEV1 $<60 \%$ predicted or RV $>200 \%$ predicted

- Bullae $>5 \mathrm{~cm}$ located in vicinity of target SPN tunnel

- Any other severe or life-threatening comorbidity that could increase the risk of bronchoscopic biopsy tunnelling, for example:

- ASA class $>3$

- >stage 3 heart failure (New York Heart Failure Classification)

- severe cachexia

- severe respiratory insufficiency or hypoxia

- Ongoing systemic infection

- Contraindication to general anaesthesia

- Participation in any other study in last 30 days

- Prior thoracic surgery on the same side of the lung as the SPN

- Pregnant or lactating

- Patients with known intrapulmonary metastases of extrapulmonary cancer/tumors

PAP, pulmonary arterial pressure; RV, right ventricel; TNM, tumor staging, tumor nodules, metastasis; TPNA, transparenchymal nodule access. 
The Archimedes System provided virtual bronchoscopy guidance to the selected POE. Once the POE location was reached, the subsequent procedural steps were adapted from the airway bypass procedure that was performed for the treatment of severe emphysema. ${ }^{17}$ A coring needle (FleXNeedle, Broncus Medical, Mountain View, California, USA) was inserted through the working channel of a standard therapeutic bronchoscope (1T180, Olympus Medical, Tokyo, Japan) and through the airway wall at the identified POE in the airway (figure 1). Aspiration was performed during slow withdrawal of the needle to further confirm that there was no vascular puncture during needle insertion. An atraumatic balloon dilator was used to enlarge the hole made at the POE, and a sheath with a blunt dissection stylet was inserted through the entry hole created in the airway. The sheath with radiopaque marker bands and a blunt dissection stylet was then used to create a tunnel within the lung parenchyma to the proximal aspect of the nodule under fused fluoroscopy guidance. The projection of the 3D target nodule from the CT model and tunnel 'guide rails' were displayed on the fused fluoroscopy image. Once the sheath tip was advanced up to $10 \mathrm{~mm}$ through the POE, the orientation of the tip was adjusted towards the target and confirmed with multiple fluoroscopy projections. Confirmation of the tunnel direction was repeated every $10 \mathrm{~mm}-20 \mathrm{~mm}$ of advancement throughout the tunnelling process. Once the proximal aspect of the nodule was reached, with sufficient clearance for opening the forceps, the stylet was removed and biopsy forceps was inserted through the sheath and biopsy samples were taken under fused fluoroscopy guidance. Where the nodules were not visible at fluoroscopy, the sheath and blunt dissection stylet were advanced to the edge of the target displayed on the fused fluoroscopy view. Furthermore, in these cases, during biopsy sampling, the patient was put on a breath-hold state each time a sample was taken to suppress the breathing motion. Additionally, since the tip of the sheath/tunnel is within close proximity of the lesion, the motion of the sheath/tunnel tip is in synchrony with the target itself.

\section{Lung resection}

After performing the BTPNA procedure, a portable chest radiograph was performed; complications, if any, were recorded, and surgical resection of the SPN was performed. The resected lobe was carefully inspected to assess the tunnel path proximity to the sampled nodule. This was verified by a pathologist. The histology from the transbronchial lung biopsies obtained at BTPNA was compared with the histology of the nodule from the resected lobe.

\section{Outcomes}

The primary endpoint of the study was to evaluate feasibility to access and biopsy the nodule by the BTPNA procedure. Safety was evaluated by carefully assessing the subject for any intraprocedural complications, such as localised bleeding, development of a pneumothorax, pneumomediastinum, or cardio-pulmonary instability. Furthermore, careful inspection of the POE into the, lung parenchyma and tunnel path was performed intraoperatively at surgical resection, and also in the resected specimen to identify any potential complications related to the BTPNA procedure. Outpatient follow-up was conducted at 90 days and 180 days to detect any subsequent serious adverse events. The severity of adverse events was defined according to the ISO 14155:2011 definition.

Feasibility was defined as the proportion of subjects in whom the procedure could be completed with obtainment of at least one biopsy sufficient for a histological diagnosis divided by the
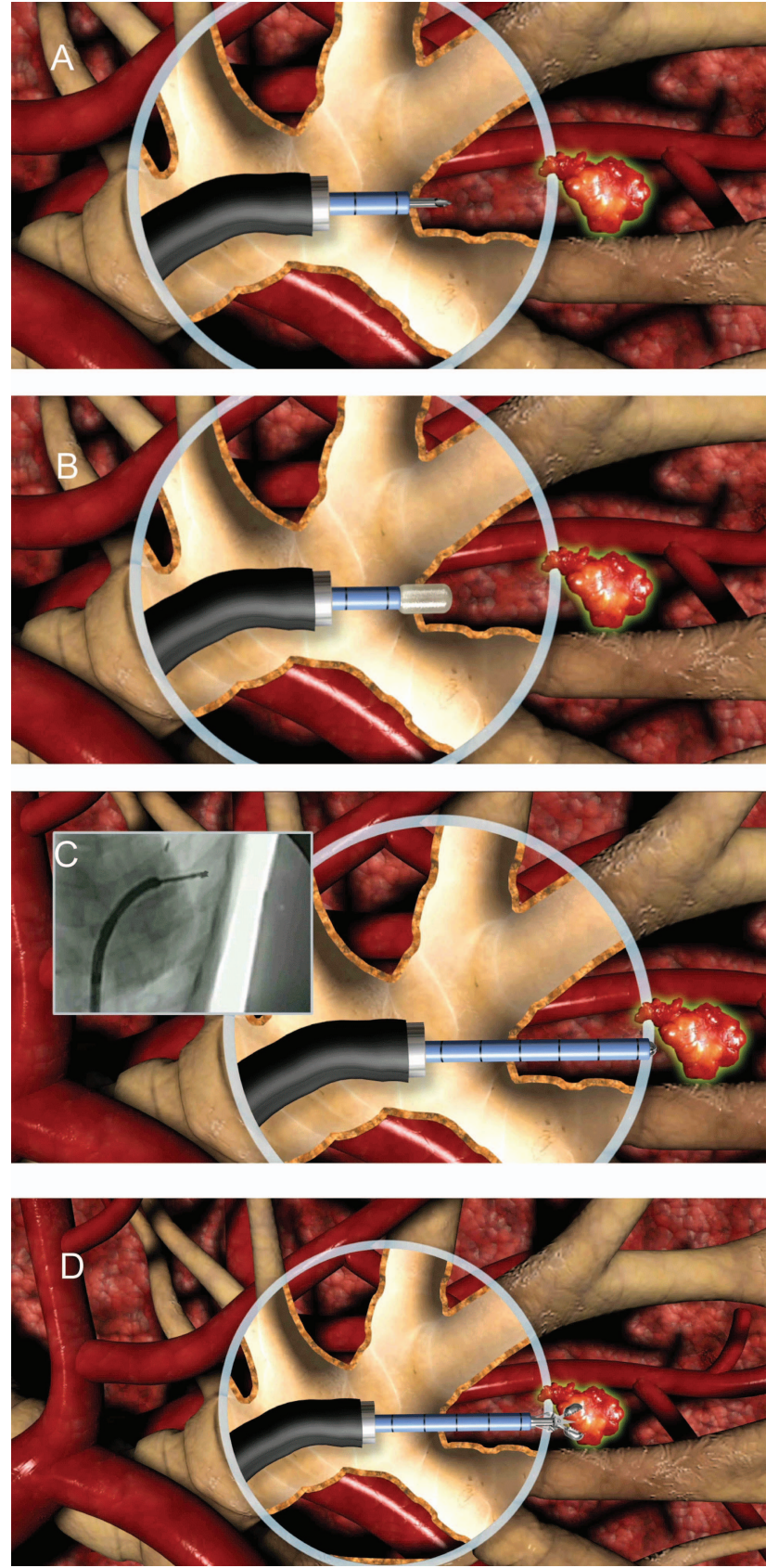

Figure 1 Diagrammatic representation of the Bronchoscopic Trans-Parenchymal Nodule Access Procedure (A) creating a hole in the airway wall at the point of entry with a needle; (B) dilating the hole with the balloon; (C) advancing the sheath with blunt stylet through parenchymal tissue in a straight line path to the nodule under fused fluoroscopic guidance provided by the system; (D) accessing, sampling, or treating the nodule with standard $2 \mathrm{~mm}$ bronchoscopic tools.

number of subjects in whom at least one attempt for BTPNA was made. Further information was obtained by inspecting the resected lobe to assess the tunnel path and its proximity to the SPN. The histology from the biopsy forceps samples was also correlated with that of the nodule from the resected lobe.

Secondary endpoints included parameters such as the biopsy yield, defined as the number of nodules with at least one biopsy sufficient for a tissue diagnosis divided by the number of nodules sampled. Other parameters evaluated included procedural time points, length of tunnel path created, number and adequacy of the biopsy samples. 


\section{Statistical analysis}

Continuous outcomes are summarised with mean, SD and other relevant statistical summaries. Where the outcomes were not normally distributed, medians and quartiles are reported. Changes in continuous variables are assessed with paired or unpaired Student's $t$ tests, as appropriate. Binary and ordinal outcomes were tabulated. Rates were expressed as percentages, and CIs for proportions were calculated using the exact binomial distribution.

This is a first in human feasibility study and, hence, we did not perform a formal power calculation. Twelve subjects undergoing biopsy via the transparenchymal sampling procedure using the Archimedes platform have provided pilot information on overall safety and feasibility.

\section{RESULTS}

Twelve patients were enrolled in this study (6 men and 6 women) with a median age of 62 years (range 40-72). The BTPNA procedure was successfully completed in 10 patients $(83 \%)$, and a positive biopsy was successfully obtained in all 10 cases in which a tunnel was successfully created from the POE to the SPN. The key procedural steps from a BTPNA procedure are shown in figure 2. There were no significant intraprocedural adverse events (table 1). Fluoroscopy, after completion of the procedure, did not reveal any occurrence of adverse events, such as pneumothorax. Inspection during surgical resection and of the resected lobe did not reveal any evidence of localised haemorrhage, bronchial or pulmonary lacerations. The only adverse event observed was a transient rise in troponin levels in one patient post-BTPNA and surgical resection. Outpatient follow-up conducted at 90 days and 180 days after the procedure did not reveal any adverse events attributable to the procedure.

Adequate histological sampling sufficient for a histological diagnosis was successfully achieved in 10 of the patients. Inspection at surgery and in the postresection specimens demonstrated that the tunnel path was created in the correct position and orientation that led directly to the target nodule. Figure 3 demonstrates the tract created in one of the patients. Furthermore, the histological findings from the biopsies obtained by BTPNA correlated with the final pathology in all the surgically resected nodules (figure 4). In the two patients where the BTPNA procedure could not be completed, the nodules were located in the apical section of the left upper lobe. In these patients, it was not possible to orientate the bronchoscope at the POE location to achieve a straight-line tunnel path to the target nodule due to limitations of the bronchoscope size and angulation, and proximity of the aorta and pulmonary artery. Furthermore, alternative access points were not practical due to the close proximity of major blood vessels. However, we were able to access nodules in the left upper lobe using the BTPNA approach in two other patients. In this initial study, there were no patients with a nodule in the right upper lobe, and the other nodules were distributed throughout the lung.

In seven of the 12 patients, the nodules were not visible at fluoroscopy. Despite this, using Archimedes fused fluoroscopy guidance, the procedure was successfully completed in six of these seven patients. In the one patient where the procedure was not completed, the main factor was the difficulty in finding a vessel-free path. Both histological confirmation and postresection evaluation of the tract confirmed accurate performance of the BTPNA procedure.
The mean procedure time was $39.8 \mathrm{~min}$, with the planning stage accounting for 18.0 (range 10.0-30.0) $\mathrm{min}$, and the creation of the tunnelled pathway 21.8 (range 12.0-40.0) $\mathrm{min}$ (table 1). The fluoroscopy time was 5.5 (range 1.8-11.6) min. The mean tunnel length was 47 (range 10-90) $\mathrm{mm}$. The overall yield for BTPNA was $83 \%$ (10 out of 12) as the tract could not be created in two patients, as described above. The biopsy yield
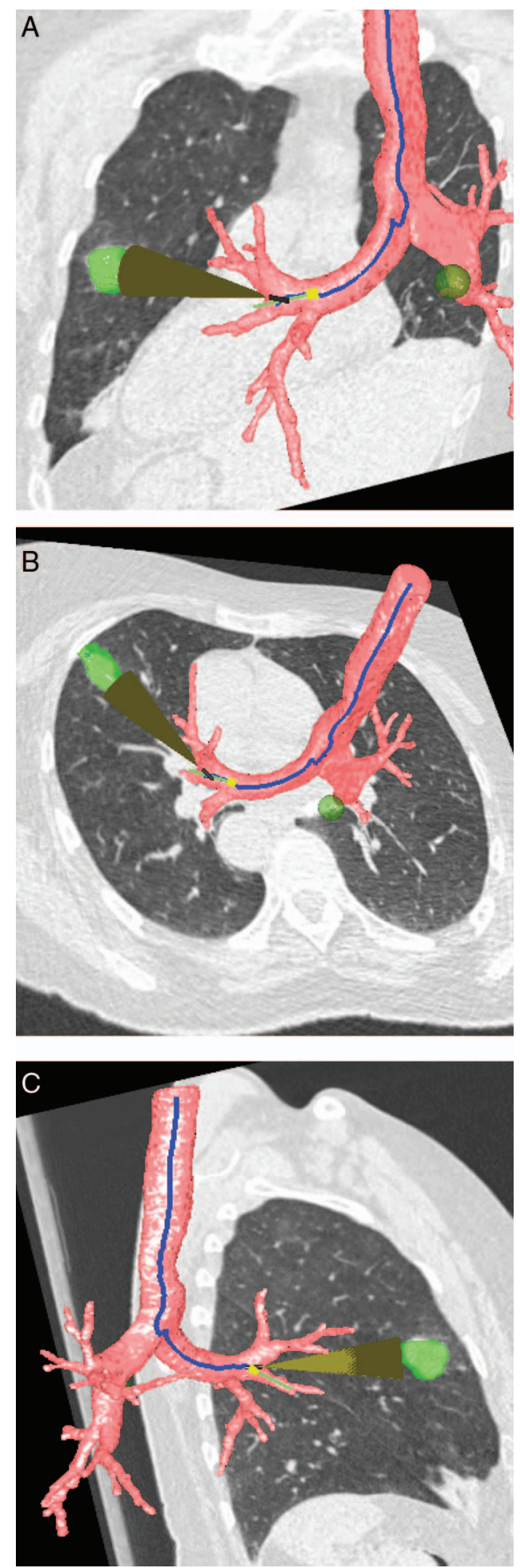

Figure 2 Three-dimensional reconstruction of thoracic CT with the bronchial tree, tunnel path, and nodule location (green highlight) located in the left upper lobe demonstrated. (A) coronal reconstruction with posterior view; (B) cross-sectional view; (C) sagittal view. 


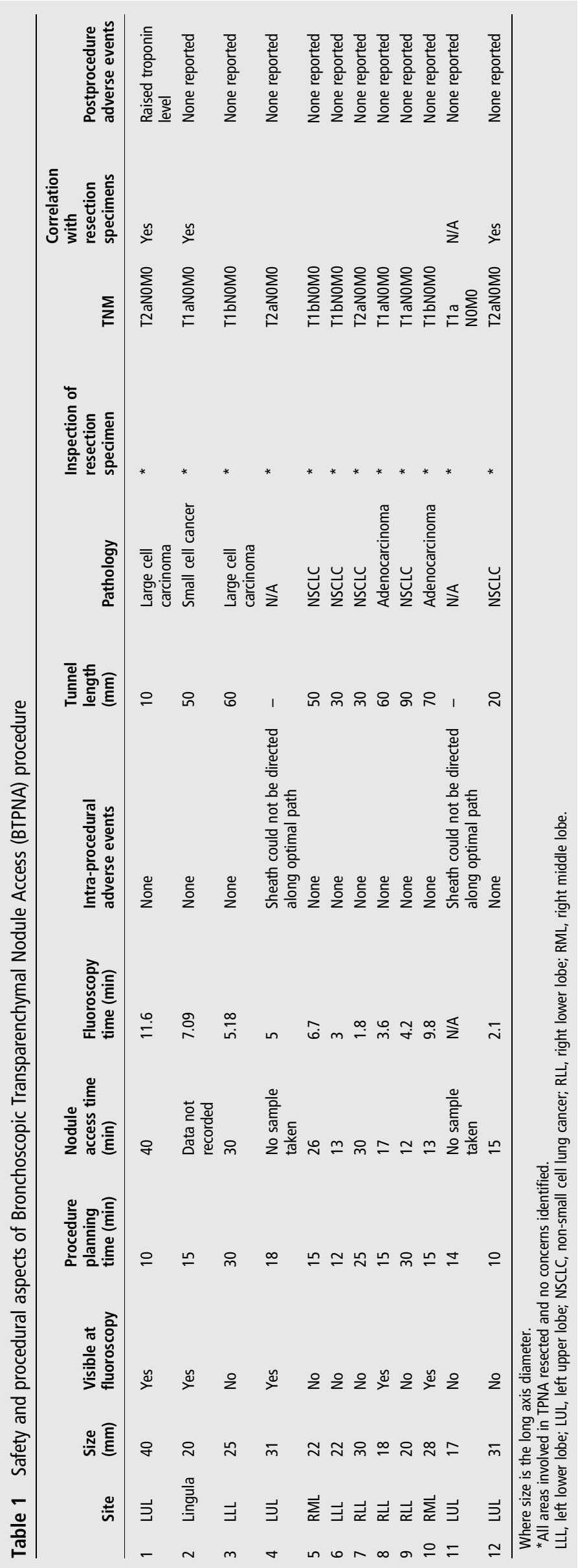

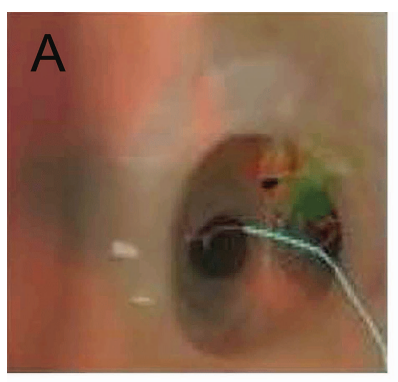
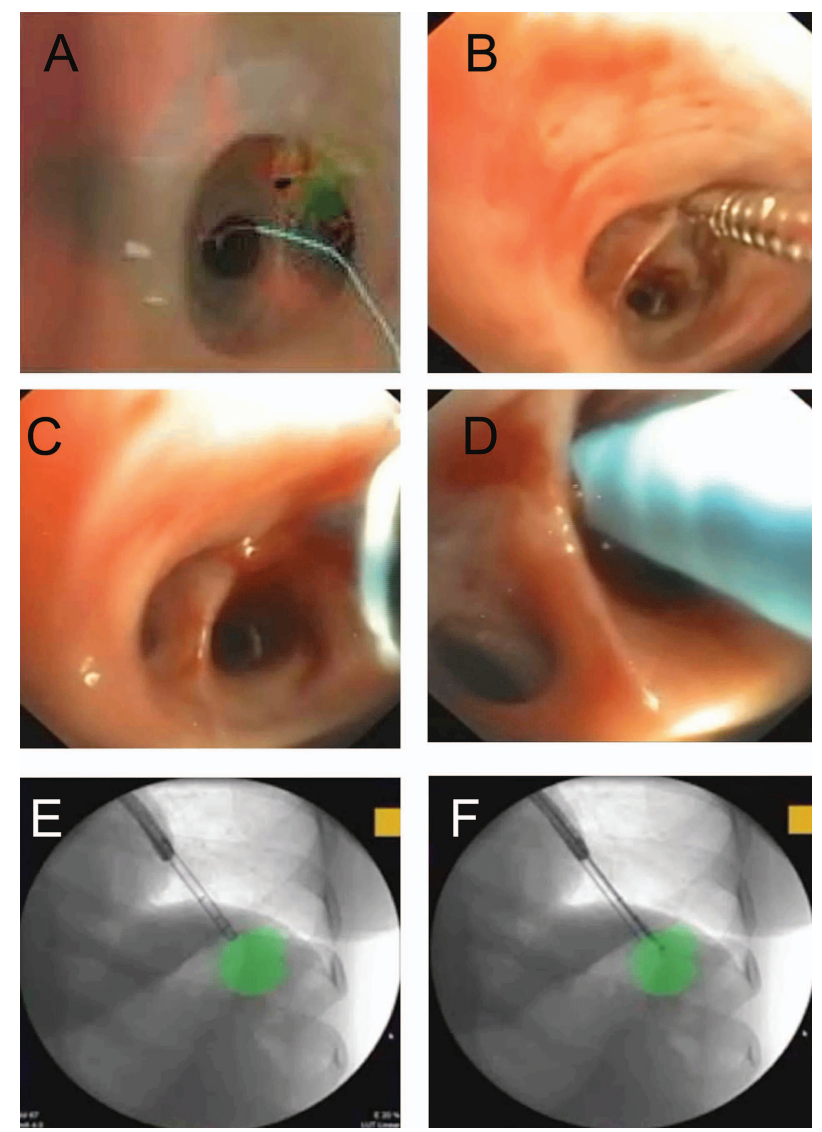

Figure 3 Bronchoscopic Trans-Parenchymal Nodule Access Procedure. (A) Archimedes Virtual Bronchoscopy Navigation System LungPoint planning module with point of entry (POE) highlighted in green;

(B) bronchoscopy view of needle insertion at POE; (C) bronchoscopy view of balloon dilatation at POE; (D) bronchoscopic view of sheath inserted in the POE; (E) fluoroscopic view of insertion of blunt dissection tip and sheath with overlay of target in green; (F) fluoroscopic view of forceps biopsy of SPN with overlay of target in green.

was $100 \%$ for procedures where a tunnelled pathway was created, and at least one biopsy attempted. An average of 3.3 biopsy samples were obtained (range 0-6) in the 12 patients who underwent BTPNA.

\section{DISCUSSION}

This report is a first in human study that evaluates the safety and feasibility of a novel approach for the access to and diagnosis of SPNs. Although the concept of creating access from the bronchial tree to the lung parenchyma has been explored in patients with severe emphysema, ${ }^{17}$ this is the first human report of creating tunnelled paths from the bronchial segments through the lung parenchyma directly to the SPNs. The procedure was successfully performed in 10 out of 12 patients with no significant adverse events observed at the time of the procedure or in the follow-up period. There was no evidence of significant immediate bleeding or pneumothoraces, which were the primary safety concerns for this approach. Clinical follow-up at 6 months did not discover any unanticipated adverse events. It was challenging to evaluate the postprocedure safety in this study as the patients proceeded directly to surgical resection. However, this provided a unique opportunity to evaluate the tunnel paths created and inspect the resected lung for any lacerations or bleeding in the surrounding lung parenchyma. The current standard of transthoracic 

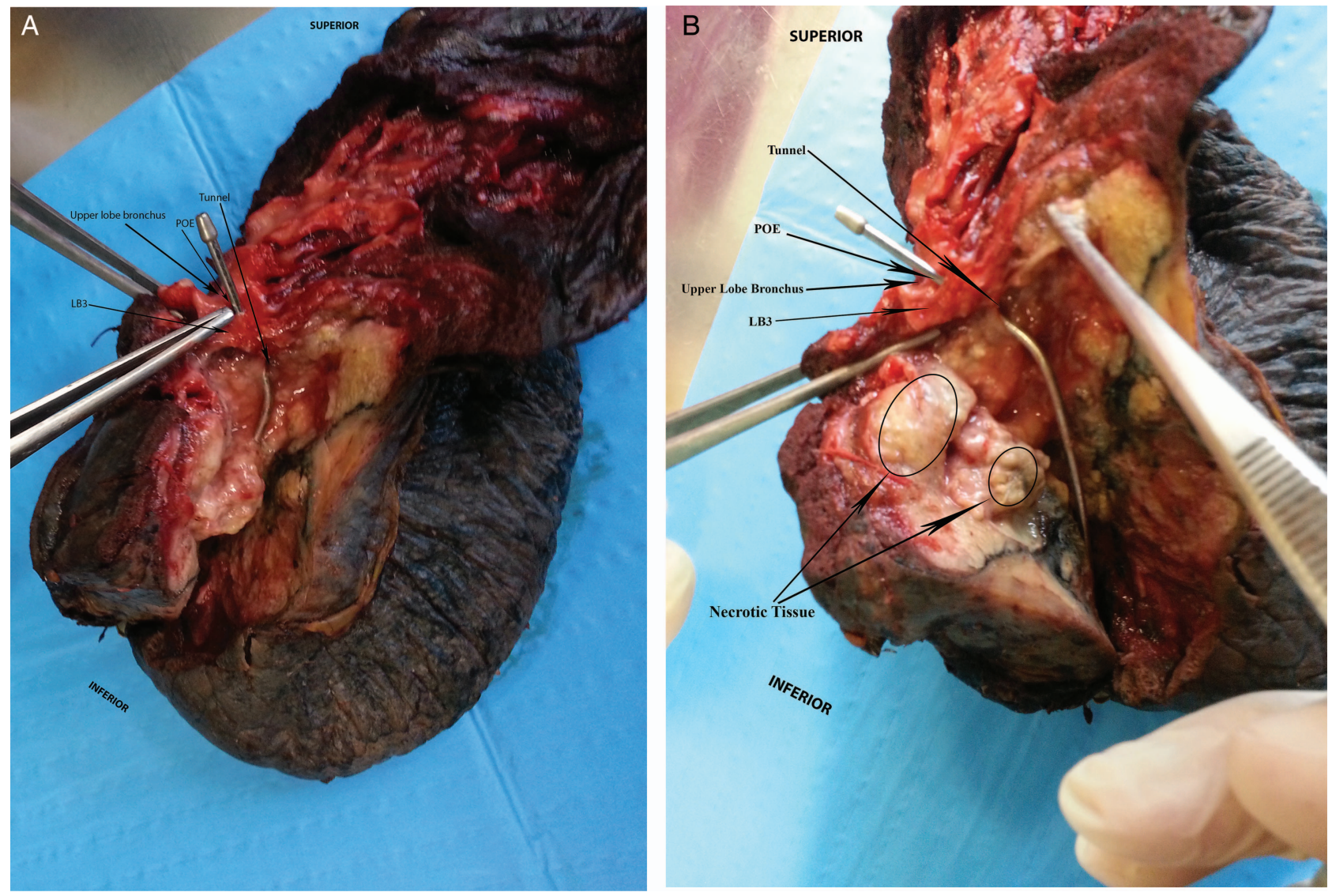

Figure 4 (A) Resected lung demonstrating the point of entry (POE) and tunnel path to the tumour located in the left upper lobe; (B) close-up view of left upper lobe and dissection to demonstrate POE in left upper lobe bronchus, tunnel path to tumour.

CT-guided biopsy appears to have a pneumothorax risk of $15 \%$ and serious bleeding risk of $1 \%{ }^{7}$

The creation of a tunnelled path and the use of a sheath enabled multiple biopsies to be performed with minimal additional risk. In this study, an average of 3.3 (range 0-6) biopsies was performed, with at least four biopsies performed in eight participants, with no evidence of increased complications with greater biopsy number. By contrast, for transthoracic needle biopsies, the number of needle insertions is associated with a greater risk of pneumothoraces. ${ }^{18}$ This increased pneumothorax risk is primarily related to the number of times the needle passes through the pleura, whereas with BTPNA, this risk is avoided. ${ }^{19}$ Furthermore, with CT-guided biopsy, the depth of needle insertion, or the distance between the pleura and the nodule, is related to both an increased risk of a pneumothorax and also the need for chest tube placement. ${ }^{20}$ In this early report with BTPNA, there was no increased risk of adverse events irrespective of the tunnel length created, despite the fact that lengths of $50 \mathrm{~mm}$ or greater were created in seven patients. In one patient, a $90 \mathrm{~mm}$ long tunnelled pathway was safely created and a biopsy successfully performed. The current limitation of BTPNA appears to be the apex of the lung where it is more difficult to control the angulation and orientation of the sheath and dissection tip in the required direction, particularly in the apex of the left upper lobe which is blocked from access by the aorta and pulmonary artery. The true extent of this limitation will become clearer with greater experience in this procedure.

The procedure may appear more complicated than a transthoracic CT-guided biopsy, but this early feasibility study appears to have a lower risk of immediate adverse events. A learning curve was demonstrated with the first four, second four and third four tunnel creation times averaging 35.0, 21.5 and $13.3 \mathrm{~min}$, respectively. Hence, after only eight procedures, the procedure time of BTPNA is comparable to either transthoracic CT-guided biopsy or standard transbronchial biopsy. BTPNA may have a complimentary role with transthoracic needle aspiration, as the former may be a more appropriate choice for nodules or tumours in certain anatomical locations, for example, centrally located nodules or behind the scapula, or where two pleural planes need to be traversed for a transthoracic approach. It may also be a safer approach in patients with emphysema due to the lower risk of pneumothorax. About a third of patients with potentially curable disease do not undergo surgical resection due to factors, such as disease, comorbidity and age. ${ }^{21}$ In the future, our approach may facilitate local treatment of early lung cancers in this group of patients.

In conclusion, the BTPNA approach improves the accessibility of peripheral nodules via the bronchoscopic route. In this small pilot study, the technique appears to be safe and feasible. Further research is warranted with multicentre interventional studies and, ultimately, a randomised controlled study against the current standard of transthoracic lung biopsy.

Twitter Follow Pallav Shah at @BromptonLungDoc

Contributors The Archimedes platform and tools were developed by Broncus Medical. The authors and Broncus Medical designed the clinical trial. FJFH and RE performed all the procedures. HH performed the surgery. PLS wrote the first draft, and all the authors contributed to the subsequent drafts. FJFH is guarantor of the publication. All authors had full access to the data. All authors agreed to the final draft and decision to submit the paper for publication. 
Funding Broncus was the sponsor and funding source of the study, and provided the Archimedes platform, FleXNeedle, balloon, and blunt dissection sheath used in the procedure. The trial was designed by an investigational study board in conjunction with the sponsor.

Competing interests The sponsor of the study (Broncus) reimbursed all clinical trial expenses incurred by the study centre. FJFH, DS, GAS and PLS have acted as consultants for Broncus Medical.

Ethics approval The study was approved by an independent ethics committee (University of Heidelberg). Subjects were recruited from March 2013 to September 2013. This study is registered with clinicaltrials.gov (NCT02130115).

Provenance and peer review Not commissioned; internally peer reviewed.

Data sharing statement All available data has been included in this manuscript.

\section{REFERENCES}

1 Lozano R, Naghavi M, Foreman K, et al. Global and regional mortality from 235 causes of death for 20 age groups in 1990 and 2010: a systematic analysis for the Global Burden of Disease Study 2010. Lancet 2012;380:2095-128.

2 Ferlay J, Shin HR, Bray F, et al. GLOBOCAN 2008 v1.2, Cancer Incidence and Mortality Worldwide: IARC CancerBase No. 10 [Internet]. Lyon, France: International Agency for Research on Cancer, 2010. http://globocan.iarc.fr

3 Paz-Ares L, Corral J. Treatment for early-stage lung cancer: what next? Lancet 2014;383:1528-30.

4 Aberle DR, Adams AM, Berg CD, et al. National Lung Screening Trial Research Team. Reduced lung-cancer mortality with low-dose computed tomographic screening. N Engl J Med 2011;365:395-409.

5 Field JK, Oudkerk M, Pedersen JH, et al. Prospects for population screening and diagnosis of lung cancer. Lancet 2013;382:732-41.

6 Wang Memoli JS, Nietert PJ, Silvestri GA. Meta-analysis of guided bronchoscopy for the evaluation of the pulmonary nodule. Chest 2012;142:385-93.

7 Wiener RS, Schwartz LM, Woloshin S, et al. Population-based risk for complications after transthoracic needle lung biopsy of a pulmonary nodule: an analysis of discharge records. Ann Intern Med 2011;155:137-44.
8 Lai RS, Lee SS, Ting YM, et al. Diagnostic value of transbronchial lung biopsy under fluoroscopic guidance in solitary pulmonary nodule in an endemic area of tuberculosis. Respir Med 1996;90:139-43.

9 Yamamoto S, Ueno K, Imamura F, et al. Usefulness of ultra- thin bronchoscopy in diagnosis of lung cancer. Lung Cancer 2004;46:43-8.

10 Asano F, Matsuno Y, Shinagawa N, et al. A virtual broncho- scopic navigation system for pulmonary peripheral lesions. Chest 2006;130:559-66.

11 Herth FJ, Ernst A, Becker HD. Endobronchial ultrasound-guided transbronchial lung biopsy in solitary pulmonary nodules and peripheral lesions. Eur Respir J 2002;20:972-4

12 Eberhardt $\mathrm{R}$, Anantham $\mathrm{D}$, Herth $\mathrm{F}$, et al. Electromagnetic navigation diagnostic bronchoscopy in peripheral lung lesions. Chest 2007;131:1800-5.

13 Eberhardt R, Anantham D, Ernst A, et al. Multimodality bronchoscopic diagnosis of peripheral lung lesions: a randomized controlled trial. Am J Respir Crit Care Med 2007; 176:36-41.

14 Eberhardt R, Ernst A, Herth FJ. Ultrasound-guided trans-bronchial biopsy of solitary pulmonary nodules less than $20 \mathrm{~mm}$. Eur Respir J 2009;34:1284-7.

15 Gaeta M, Pandolfo I, Volta S, et al. Bronchus sign on CT in peripheral carcinoma of the lung: value in predicting results of transbronchial biopsy. AJR Am J Roentgenol 1991; 157:1181-5.

16 Shah PL. Atlas of bronchoscopy. UK: Hodder Arnold, 2011.

17 Shah PL, Slebos DJ, Cardoso PFG, et al. Bronchoscopic lung volume reduction with exhale airway stents for emphysema (EASE trial): randomised, sham-controlled multicentre trial. Lancet 2011;378:997-1005.

18 Froelich JJ, Ishaque N, Regn J, et al. Guidance of percutaneous pulmonary biopsies with real-time CT fluoroscopy. Eur J Radiol 2002;42:74-9.

19 Ohno Y, Hatabu H, Takenaka D, et al. CT-guided transthoracic needle aspiration biopsy of small ( $\leq 20 \mathrm{~mm}$ ) solitary pulmonary nodules. AJR 2003;180:1665-9.

20 Hiraki T, Mimura H, Gobara $\mathrm{H}$, et al. Incidence of and risk factors for pneumothorax and chest tube placement after CT fluoroscopy-guided percutaneous lung biopsy: retrospective analysis of the procedures conducted over a 9-year period. AJR Am J Roentgenol 2010;194:809-14.

21 Cykert S, Dilworth-Anderson P, Monroe MH, et al. Factors associated with decisions to undergo surgery among patients with newly diagnosed early-stage lung cancer. JAMA 2010;303:2368-76. 